für $\omega_{\mathrm{p}}=10^{16} \mathrm{sec}^{-1}, v_{0} \sim 1 \cdot 4 \cdot 10^{8} \mathrm{~cm} \mathrm{sec}^{-1}$ und $c_{\mathrm{s}}=2 \cdot 10^{5} \mathrm{~cm} \mathrm{sec}^{-1}$.

Es findet also im allgemeinen bei $\lambda \sim \delta$ keine ausgeprägte Verminderung der Absorption statt, sondern das Verhalten des Absorptionskoeffizienten bei $\omega \approx \omega_{0}$ hängt empfindlich von der Schallgeschwindigkeit ab. Für $\omega \gg \omega_{1}$ setzt eine starke Abnahme der Absorption ein.

Natürlich ist das Matrixelement (21) auch für longitudinale Wellen von Null verschieden und die Berechnung des entsprechenden Anteils an der longitudinalen Absorption kann in ganz analoger Weise berechnet werden. Man findet

$$
\alpha_{\| \text {mag }}=2 \frac{c_{\text {long }} \cdot c_{\text {trans }}}{v_{0}{ }^{2}} \alpha_{\perp \text { mag }} .
$$

Man kann also bei der Berechnung der Absorptions- koeffizienten für longitudinale Wellen den Anteil der magnetischen Kopplung vernachlässigen.

Interessant ist es jedoch, für $\lambda \ll \delta$ und $\lambda \gg \delta$ das Verhältnis $\alpha_{\perp \mathrm{mag}} / \alpha_{\| \mathrm{el}} \mathrm{zu}$ betrachten. Man findet

$$
\frac{\alpha_{\perp \mathrm{mag}}}{\alpha_{\| \mathrm{el}}}=\left\{\begin{array}{l}
\frac{8}{\pi^{2}}\left(\frac{c_{\text {long }}}{c_{\text {trans }}}\right)^{2} \quad(\lambda \gg \delta), \\
\frac{9}{2}\left(\frac{\omega_{\mathrm{p}}}{\omega}\right)^{4}\left(\frac{c_{\text {trans }}}{c}\right)^{4}\left(\frac{c_{\text {long }}}{v_{0}}\right)^{2} \quad(\lambda \ll \delta) .
\end{array}\right.
$$

Solange $\lambda \ll \delta$ ist, sind also die Absorptionskoeffizienten für transversalen bzw. longitudinalen Ultraschall etwa von der gleichen Größenordnung. Für $\lambda \gg \delta$ nimmt die transversale Absorption stark ab.

Abschließend danke ich Herrn J. Mertsching für die kritische Durchsicht des Manuskriptes und zahlreiche klärende Bemerkungen.

\title{
Die Hauptdielektrizitätskonstanten der homogen geordneten kristallinflüssigen Phase des $p$-Azoxyanisols
}

\author{
Von W. MaIER und G. Meier \\ Aus dem Institut für physikalische Chemie und dem Physikalischen Institut der Universität \\ Freiburg i. Br. \\ (Z. Naturforschg. 16 a, 470-477 [1961] ; eingegangen am 25. Februar 1961)
}

\begin{abstract}
An hochgereinigtem 4,4'-Di-methoxy-azoxybenzol ( $p, p^{\prime}$-Azoxyanisol) wurden die Hauptdielektrizitätskonstanten $\varepsilon_{1}$ und $\varepsilon_{2}$ und die dielektrische Anisotropie $\Delta \varepsilon$ der nematischen kristallinflüssigen Phase bei 0,$10 ; 0,26 ; 0,65$ und 1,60 MHz gemessen. Die Ergebnisse werden mit der in einer früheren Veröffentlichung entwickelten Theorie verglichen. Zur Auswertung werden die molekularen Hauptpolarisierbarkeiten aus Brechungsindexmessungen von Chatelain berechnet und damit das Dipolmoment und seine Orientierung im Molekül aus den DK-Daten bestimmt.
\end{abstract}

Um die in der vorangegangenen Veröffentlichung ${ }^{1}$ aufgestellte molekulare Theorie der Hauptdielektrizitätskonstanten $\varepsilon_{1}$ und $\varepsilon_{2}$ einer homogen geordneten nematischen Phase an der meistuntersuchten Substanz dieser Art, dem $p$-Azoxyanisol (=4,4' -Dimethoxy-azoxybenzol), prüfen zu können, haben wir diese Größen mit einer verbesserten Apparatur an besonders hochgereinigter Substanz nochmals gemessen und sie nach den früher ${ }^{1}$ angegebenen theoretischen Beziehungen ausgewertet. Die erneute Messung von $\varepsilon_{1}$ und $\varepsilon_{2}$ schien uns wünschenswert, nachdem unsere früheren Untersuchungen ${ }^{2}$ merklich höhere $\varepsilon$-Werte ergeben hatten, als sie von anderen Autoren berichtet worden waren.

1 W. Maier u. G. Meier, Z. Naturforschg. 16 a, 262 [1961].

\section{Experimenteller Teil}

a) Substanz: p-Azoxyanisol der Fa. Th. S chu$\mathrm{ch}$ a rd t, München, wurde aus einem Methanol-BenzolGemisch umkristallisiert und chromatographisch auf Reinheit geprüft. Die zur Messung benutzte Substanz hatte einen Schmelzpunkt von $118,0^{\circ} \mathrm{C}$ und einen Klärpunkt von $135,3^{\circ} \mathrm{C}$ (Schmelzpunktsröhrchen, mit Fadenkorrektur). Ihr Klärpunkt liegt höher als der der früher untersuchten Präparate und ebenso ergaben sich auch größere Werte für die dielektrische Anisotropie $\Delta \varepsilon$. Beides spricht für höhere Reinheit der hier untersuchten Substanz.

b) Apparatur: Die Dielektrizitätskonstante (DK) wurde bei den Frequenzen 0,$10 ; 0,26 ; 0,65$ und 1,60 $\mathrm{MHz}$ mit Hilfe des Multidekameters DK 06 (Sonderausführung) der Fa. Wissenschaftlich-technische Werk-

2 W. MaIer, G. B ARth u. H. E. Wiehl, Z. Elektrochem. 58, 674 [1954]. - W. MAIER, Z. Naturforschg. 2 a, 458 [1947]. 
stätten Weilheim/Obb. nach der Substitutionsmethode bestimmt. Mit demselben Gerät konnten auch die Verluste $\operatorname{tg} \delta$ nach einer Resonanzmethode gemessen werden.

Der entscheidende Teil der Apparatur, die Meßzelle, stellt eine Weiterentwicklung der früher ${ }^{2}$ benutzten Form dar. Letztere hatte den Nachteil starker Temperaturabhängigkeit und mangelhafter Reproduzierbarkeit der Leerkapazität. Die Neukonstruktion besteht aus drei kreisförmigen Palladiumplatten (thermischer Ausdehnungskoeffizient $\left.\beta=10,6 \cdot 10^{-6} \operatorname{grad}^{-1}\right)$, die mit zwei Abstandsringen aus Frequenta ${ }^{*}\left(\beta=8 \cdot 10^{-6}\right)$ zu einem Doppelplattenkondensator zusammengelötet sind [Plattenabstand 2,7 mm; Nutzkapazität (Luft) $6,3 \mu \mathrm{F}$; Substanzbedarf $\sim 6 \mathrm{~cm}^{3}$. Die Meßzelle ist von einem Thermostatenmantel (angeschlossen an Umlaufthermostat) umgeben. Im Bereich von 70 bis $140{ }^{\circ} \mathrm{C}$ ist ihre zeitliche Temperaturkonstanz besser als $\pm 1 / 100^{\circ}$; die maximalen Temperaturdifferenzen innerhalb der Meßzelle liegen unter $1 / 10^{\circ}$. Zur einheitlichen Orientierung der nematischen Flüssigkeit (makroskopische Orientierung durch Beseitigung aller von Konvektionsströmungen usw. verursachten Deformationen) befindet sich der Meßraum des Kondensators in einem homogenen Ma-

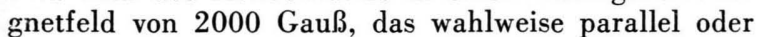
senkrecht zur Normalen der Kondensatorplatten gestellt werden kann. Wie schon früher ${ }^{2}$ beobachtet worden war, ist die angegebene Magnetfeldstärke bei weitem ausreichend, um in einer Meßzelle mit guter Temperaturhomogenität eine vollständig homogene Orientierung der hier untersuchten nematischen Phase zu erzielen. Die Feldstärke des elektrischen Meßwechselfeldes lag für die $\varepsilon$-Messungen bei etwa $100 \mathrm{Volt} / \mathrm{cm}$, für die $\operatorname{tg} \delta$-Messungen bei etwa $11 \mathrm{Volt} / \mathrm{cm}$. Die aus der dielektrischen Anisotropie der nematischen Phasen entspringende orientierende Wirkung des elektrischen Meßwechselfeldes vermag bei diesen Feldstärken die magnetische Orientierung noch nicht merklich zu stören, wie sich durch DK-Messungen bei verschiedenen Magnetfeldstärken leicht kontrollieren ließ. $\varepsilon$ wird aus der Kapazitätsdifferenz zwischen der mit der Substanz gefüllten und der luftgefüllten Meßzelle bestimmt. Hierzu ist eine Eichung mit einer Flüssigkeit bekannter DK und die experimentelle Bestimmung einer frequenzabhängigen Korrekturgröße notwendig, die den Einfluß des Wechselstromwiderstandes der Verbindungsleitung zwischen Meßzelle und Meßkondensator bei den höheren Frequenzen berücksichtigt. Die Eichung wurde mit Benzol im Temperaturbereich von 20 bis $70{ }^{\circ} \mathrm{C}$ durchgeführt (Benzol, p. a., von $\mathrm{R}$ i e d e l d e $\mathrm{H}$ a e $\mathrm{n}$, mehrere Tage über Natrium destilliert, $\varepsilon_{20^{\circ}}=2,2836$, $\left.\mathrm{d} \varepsilon / \mathrm{d} t=-0,00190^{3}\right)$. Die Temperaturabhängigkeit des Eichfaktors ist sehr gering und außerdem exakt linear, so daß die Extrapolation bis $140{ }^{\circ} \mathrm{C}$ keine Schwierigkeit bereitet. Die Änderung von 20 bis $140^{\circ} \mathrm{C}$ beträgt nur 2,5\% und liegt damit noch unter der von der Unsicherheit des Absolutwertes der Eichflüssigkeit herrüh-

* Wir sind der Firma Steatit Magnesia A.G., Herrn Dr. Lufr, für ihre entgegenkommende Beratung und die Erprobung verschiedener Materialien zu bestem Dank verbunden. renden Ungenauigkeit. Letztere dürfte - insbesondere bei höheren Temperaturen - bei $\pm 3 \%$ liegen. Die Leitungskorrektur konnte mit Benzol-Fluorbenzol-Mischungen bekannter DK genügend genau bestimmt werden.

c) Ergebnisse: Wie in den früheren Arbeiten ${ }^{2}$ bezeichnen wir die in Richtung der Achse der nematischen Ordnung (parallel zum Magnetfeld) gemessene DK mit $\varepsilon_{1}$, die senkrecht dazu gültige mit $\varepsilon_{2}$.

Abb. 1 bringt die Ergebnisse für $v=650 \mathrm{kHz}$. Die $\varepsilon$-Werte haben absolut (mit Eichfehler) eine Genauigkeit von $\pm 0,5 \%$; der für die Temperaturund Frequenzabhängigkeit allein maßgebende Streufehler ist kleiner als $0,1 \%$. Die Anisotropiegröße $\Delta \varepsilon \equiv\left(\varepsilon_{1}-\varepsilon_{2}\right)$ kann aus der bei $90^{\circ}$-Drehung des Magnetfelds auftretenden Kapazitätsänderung direkt bestimmt werden. Diese $\Delta \varepsilon$-Werte sind mit $\pm 1 \%$ genauer als die durch die Differenzbildung $\varepsilon_{1}-\varepsilon_{2}$ erhaltenen und in Abb. 1 gesondert eingezeichnet.

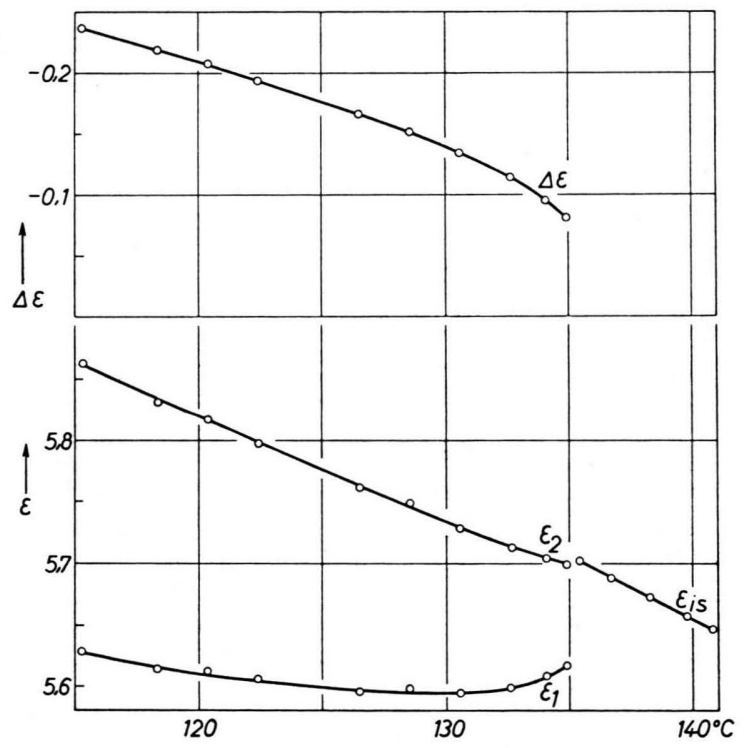

Abb. 1. p-Azoxyanisol. Die Dielektrizitätskonstanten $\varepsilon_{1}, \varepsilon_{2}$, $\varepsilon_{\text {is }}$ und die dielektrische Anisotropie $\Delta \varepsilon=\varepsilon_{1}-\varepsilon_{2}$.

Die bei 100 und $260 \mathrm{MHz}$ sowie bei $1,6 \mathrm{MHz}$ erhaltenen Werte liegen im allgemeinen innerhalb der Fehlergrenzen auf den Kurven der Abb. 1. Nur im Temperaturbereich unterhalb $125^{\circ} \mathrm{C}$ treten bei $\varepsilon_{1}$ geringe systematische Differenzen auf, welche die Streufehlergrenzen knapp übersteigen und ein $\mathrm{Ab}$ sinken von $\varepsilon_{1}$ mit wachsender Frequenz andeuten,

3 L. Hartshorn, J. V. L. Parry u. L. Essen, Proc. Phys. Soc., Lond. B 68, 422 [1955]. 
also den Beginn einer $\varepsilon_{1}$-Dispersion, wie sie nach der Theorie ${ }^{1}$ erwartet werden kann und bei den höheren Homologen des Azoxyanisols auch tatsächlich zu beobachten ist ${ }^{4}$.

Die Verlustmessungen ergaben für $\varepsilon_{2}^{\prime \prime}$ zeitlich variable, mangelhaft reproduzierbare Werte von der Größenordnung $10^{-3}$ bis $10^{-2}$, die auf Ionenverunreinigungen zurückzuführen sind, da sie mit wachsender Frequenz abnahmen. $\varepsilon_{1}{ }^{\prime \prime}$ war deutlich größer als $\varepsilon_{2}{ }^{\prime \prime}$ und nahm, insbesondere bei den tieferen Temperaturen, mit steigender Frequenz zu. Es liegt hier neben den Ionenleitfähigkeitsverlusten also noch ein Verlustanteil entgegengesetzter Frequenzabhängigkeit vor, der uns die beginnende $\varepsilon_{1}$-Dispersionsstufe anzudeuten scheint; doch waren die erhaltenen Werte ebenfalls kaum reproduzierbar und damit für eine quantitative Auswertung ungeeignet.

Mit den früher ${ }^{2}$, ebenfalls bei $650 \mathrm{kHz}$ gemessenen Werten verglichen, liegen die neuen $\varepsilon$-Werte um 1 bis $2 \%$, die $\Delta \varepsilon$-Werte um 5 bis $8 \%$ höher. Die Vergrößerung der Anisotropie zeigt deutlich, daß die Unterschiede auf die höhere Reinheit der Substanz (vgl. oben) zurückzuführen sind.

Die $\Delta \varepsilon$-Kurve zeigt einen im großen und ganzen linearen Temperaturverlauf, jedoch ein stärkeres Absinken bei Annäherung an den Klärpunkt. In einer früheren Arbeit ${ }^{2}$ hatten wir vermutet, daß diese Abweichungen vom linearen Temperaturverlauf durch Temperaturinhomogenitäten in der Meßzelle und uneinheitlichen Klärpunkt der durch Zersetzungsprodukte verunreinigten Substanz verursacht seien, nachdem wir an einem höheren Homologen, der entsprechenden Pentyloxy-Verbindung, eine exakt lineare Temperaturabhängigkeit von $\Delta \varepsilon$ bis zum Klärpunkt hin gefunden hatten. Die in Abb. 1 zu beobachtende Krümmung der $\Delta \varepsilon$-Kurve muß jedoch als reell angesehen werden, da wir sicher sind, daß die Temperaturdifferenzen in unserer neuen Meßzelle äußerstenfalls $0,1^{\circ}$ betragen konnten und die $\Delta \varepsilon$-Kurve sich auch nach längerem Erhitzen der Substanz exakt reproduzieren ließ (die tatsächlich auftretenden chemischen Veränderungen waren sehr geringfügig und verursachten Klärpunktsverschiebungen von nur etwa $0,1^{\circ}$; sie machten sich nur in den $\operatorname{tg} \delta$-Werten bemerkbar, nicht aber in den $\varepsilon$ - und $\Delta \varepsilon$-Werten). Der bei der Pentyl-Verbindung beobachtete exakt lineare $\Delta \varepsilon$-Verlauf ist vermutlich ein Sonderfall. Tatsächlich haben wir einen solchen bei

${ }^{4}$ W. Maier u. G. Meier (in Vorbereitung). keinem anderen Homologen gefunden, weder bei der Butyl- noch bei der Hexyl- und Heptyl-Verbindung ${ }^{4}$.

\section{Vergleich mit der Theorie und Auswertung}

Die Auswertung der $\varepsilon$ - und $\Delta \varepsilon$-Daten stützt sich auf die aus der molekularen Theorie $^{1}$ folgenden Beziehungen (1) - (4) :

$$
\begin{aligned}
\frac{\varepsilon_{\mathrm{is}}-1}{4 \pi}=N_{\mathrm{is}} h F\left\{\bar{\alpha}+F-\frac{\mu^{2}}{3 k T}\right\}, \\
\frac{\varepsilon_{1}-1}{4 \pi}=N_{\mathrm{n}} h F\left\{\bar{\alpha}+\frac{2}{3} \Delta \alpha \cdot S\right. \\
\left.+F \frac{\mu^{2}}{3 k T}\left[1-\left(1-3 \cos ^{2} \beta\right) S\right]\right\}, \\
\frac{\varepsilon_{2}-1}{4 \pi}=N_{\mathrm{n}} h F\left\{\bar{\alpha}-\frac{1}{3} \Delta \alpha \cdot S\right. \\
\left.+F \frac{\mu^{2}}{3 k T}\left[1+\frac{1}{2}\left(1-3 \cos ^{2} \beta\right) S\right]\right\}, \\
\frac{\Delta \varepsilon}{4 \pi}=N_{\mathrm{n}} h F\left\{\Delta \alpha-F \frac{\mu^{2}}{2 k T}\left(1-3 \cos ^{2} \beta\right)\right\} S
\end{aligned}
$$

Hierin bedeuten: $N_{\text {is }}=N_{\mathrm{L}} \varrho_{\text {is }} / M$ bzw. $N_{\mathrm{n}}=N_{\mathrm{L}} \varrho_{\mathrm{n}} / M$ die Moleküldichte der isotropflüssigen bzw. nematischen Phase;

$h=\frac{3 \varepsilon}{2 \varepsilon+1} ; F=\frac{1}{1-\bar{\alpha} \cdot f}$ mit $f=\frac{2 \varepsilon-2}{2 \varepsilon+1} \frac{4 \pi}{3} N_{\mathrm{L}} \frac{\varrho}{M} ;$

für $\varepsilon$ und $\varrho$ sind in der isotropflüssigen Phase die Werte $\varepsilon_{\text {is }}$ und $\varrho_{\text {is }}$, in der nematischen Phase die Werte $\bar{\varepsilon}=\frac{1}{3}\left(\varepsilon_{1}+2 \varepsilon_{2}\right)$ und $\varrho_{\mathrm{n}}$ zu verwenden; $S=$ nematischer Ordnungsgrad.

$\bar{\alpha}=\frac{1}{3}\left(\alpha_{1}+2 \alpha_{\mathrm{q}}\right) ; \Delta \alpha=\alpha_{1}-\alpha_{\mathrm{q}} ; \alpha_{1}$ ist die Hauptpolarisierbarkeit parallel zur Vorzugsachse $\zeta$ des Moleküls, $\alpha_{q}$ der Mittelwert der Querpolarisierbarkeiten. Die $\zeta$-Achse fällt annähernd mit der Moleküllängsachse zusammen und ist diejenige Molekülachse, mit der sich die Moleküle in der nematischen Phase parallel zueinander stellen. $\mu=$ Dipolmoment des freien Moleküls, $\beta=$ Winkel zwischen Dipolmoment und $\zeta$-Achse.

\section{a) Bedeutung von $\mu$ und $\beta$}

Bei der Ableitung der Beziehungen (1) bis (4) ist ein mit dem Molekül starr verbundenes, einheitliches Dipolmoment angenommen worden. Das $\vec{\mu}$ des Azoxyanisols setzt sich aber zusammen aus dem mit dem Molekülhauptteil starr verbundenen Moment $\vec{\mu}_{\mathrm{M}}$ der Azoxygruppe und den beiden Teilmomenten der um die Para-Achse der Benzolringe drehbaren Methoxy-Gruppen, deren Para-Achsen- 
Komponenten sich kompensieren, so da $\beta$ nur die senkrecht dazu stehenden Komponenten $\vec{\mu}_{\perp}$ wirksam sind. Um das dielektrische Verhalten exakt angeben zu können, sollte man das Behinderungspotential der innermolekularen Drehung der Methoxygruppen um die $\mathrm{C}_{\mathrm{ar}}-\mathrm{O}$-Bindungen kennen. Es ist sehr wahrscheinlich, daß dasselbe zwei Minima für die mit den Benzolringen coplanaren Stellungen der Methoxygruppe aufweist. Wir betrachten nun zwei Grenzfälle:

1. Die Potentialminima sind so tief, daß das Molekül praktisch in vier rotationsisomeren ebenen Formen existiert.

2. Die Potentialminima sind sehr flach, so daß praktisch freie Drehbeweglichkeit der Methoxygruppen vorliegt.

Fall 1: Die vier Rotationsisomeren sind energetisch nahezu gleichwertig; die beiden trans-Formen mit dem Gesamtmoment $\vec{\mu}_{\mathrm{M}}$ und die beiden cisFormen mit dem Gesamtmoment $\vec{\mu}_{\mathrm{M}}+2 \vec{\mu}_{\perp}$ bzw. $\vec{\mu}_{\mathrm{M}}-2 \vec{\mu} \perp$ treten also gleich häufig auf. In diesem Falle wird, wie sich durch Berechnung des Mittelwerts von $\vec{\mu}$ leicht zeigen läßt, durch die Gleichungen

$$
\mu_{\mathrm{M}}^{2} \cos ^{2} \gamma+2 \mu_{\perp}^{2} \sin ^{2} \delta=\mu^{2} \cos ^{2} \beta
$$

und $\quad \mu_{\mathrm{M}}^{2} \sin ^{2} \gamma+2 \mu_{\perp}^{2} \cos ^{2} \delta=\mu^{2} \sin ^{2} \beta$

ein dieselbe Orientierungspolarisation lieferndes molekülfestes „Ersatzmoment“ $\mu$ definiert, das mit der $\zeta$-Achse den Winkel $\beta$ bildet $(\gamma=$ Winkel zwischen $\vec{\mu}_{\mathrm{M}}$ und $\zeta$-Achse, $\delta=$ Winkel zwischen ParaAchse und $\zeta$-Achse). Die Auflösung ergibt:

$$
\begin{gathered}
\mu=\sqrt{\mu_{\mathrm{M}}^{2}+2 \mu_{\perp}^{2}}, \\
\cos ^{2} \beta=\frac{\mu_{\mathrm{M}}^{2} \cos ^{2} \gamma+2 \mu_{\perp}^{2} \sin ^{2} \delta}{\mu_{\mathrm{M}}^{2}+2 \mu_{\perp}^{2}} .
\end{gathered}
$$

Fall 2: Die entsprechenden Beziehungen bei Gleichwahrscheinlichkeit aller Stellungen der Methoxy-Gruppen und deren gegenseitigen Unabhängigkeit lauten:

$$
\begin{gathered}
\mu_{\mathrm{M}}^{2} \cos ^{2} \gamma+2 \mu_{\perp}^{2} \cdot \frac{1}{2} \sin ^{2} \delta=\mu^{2} \cos ^{2} \beta, \\
\mu_{\mathrm{M}}^{2} \sin ^{2} \gamma+2 \mu_{\perp}^{2}\left(1-\frac{1}{2} \sin ^{2} \delta\right)=\mu^{2} \sin ^{2} \beta, \\
\mu=\sqrt{\mu_{\mathrm{M}}^{2}+2 \mu_{\perp}^{2}}, \quad \cos ^{2} \beta=\frac{\mu_{\mathrm{M}}^{2} \cos ^{2} \gamma+\mu_{\perp}^{2} \sin ^{2} \delta}{\mu_{\mathrm{M}}^{2}+2 \mu_{\perp}^{2}} .
\end{gathered}
$$

Für $\mu$ erhält man also in beiden Fällen dasselbe Er-

5 W. Maier u. A. SAUPe, Z. Naturforschg. 15 a, 287 [1960]. gebnis, während sich für $\cos ^{2} \beta$ ein Unterschied ergibt, der aber wegen des kleinen Wertes von $\delta$ (s. unten) praktisch keine Rolle spielt.

Die Lage der $\zeta$-Achse im Molekül läßt sich natürlich nicht genau angeben; sie stimmt innerhalb der vorläufig erreichbaren Genauigkeiten mit der Längsachse der äußeren Molekülform überein, wobei letztere wegen der Rotationsisomerie mit einer gewissen Unsicherheit behaftet ist. Um für alle Diskussionen eine klare Basis zu schaffen, nehmen wir als $\zeta$-Achse die Verbindungslinie der Benzolringmittelpunkte, womit aus Stuart-Modellen $\delta=11^{\circ}$ folgt.

\section{b) Bestimmung der Hauptpolarisierbarkeiten}

Der untersuchte Temperaturbereich ist zu klein, als daß die Temperaturabhängigkeit der DK mit zur Auswertung herangezogen werden könnte. Wir verwenden daher nur die bei $115^{\circ} \mathrm{C}$ gemessenen Daten. Dichte $^{5}$ und Ordnungsgrad ${ }^{6}$ sind bekannt; es sind also die Unbekannten $\alpha, \Delta \alpha, \mu$ und $\beta$ zu bestimmen. Wegen $\Delta \varepsilon=\varepsilon_{1}-\varepsilon_{2}$ und

$$
\frac{\left(\varepsilon_{\text {is }}-1\right)}{\varrho_{\text {is }}}=\frac{1}{3}\left[\frac{\left(\varepsilon_{1}-1\right)}{\varrho_{\mathrm{n}}}+\frac{2\left(\varepsilon_{2}-1\right)}{\varrho_{\mathrm{n}}}\right]
$$

stehen nur zwei unabhängige Beziehungen zur Verfügung. Wir bestimmen im folgenden daher $\bar{\alpha}$ und $\alpha_{q}$ (und damit auch $\alpha_{1}$ und $\Delta \alpha$ ) aus den von ChateLAIN $^{7}$ gemessenen Brechungsindizes und können dann $\mu$ und $\beta$ aus unseren DK-Messungen mittels (1) und (4) ermitteln.

Die Elektronenanteile $\bar{\alpha}^{*}$ und $\alpha_{\mathrm{q}}{ }^{*}$ von $\bar{\alpha}$ und $\alpha_{\mathrm{q}}$ erhält man durch Extrapolation nach $\lambda=\infty$ aus den wellenlängenabhängigen $\alpha^{*}(\lambda)$, die sich aus den Brechungsindizes in folgender Weise ergeben:

$\bar{\alpha}^{*}(\lambda)$ berechnet sich aus dem Brechungsindex $n_{\text {is }}$ und der Dichte $\varrho_{\text {is }}$ der isotropflüssigen Phase nach

$$
\bar{\alpha}^{*}(\lambda)=\frac{n_{\mathrm{is}}^{2}-1}{n_{\mathrm{is}}^{2}+2} \frac{M}{\varrho_{\mathrm{is}}} \frac{3}{4 \pi N_{\mathrm{L}}} .
$$

Hier ist für das innere Feld das Lonentz-Feld angenommen.

$\alpha_{1}^{*}(\lambda)$ und $\alpha_{\mathrm{q}}{ }^{*}(\lambda)$ bestimmen wir aus den Hauptbrechungsindizes $n_{1}, n_{2}, n_{3}$ des Kristalls. Hier ist der LoRentz-Ansatz natürlich nicht mehr brauchbar. Ebenso ist die Berechnung des inneren Feldes nach Onsager nicht möglich, weil die Doppelbrechung $40 \%$ des mittleren Brechungsindex beträgt,

\footnotetext{
6 A. Saupe u. W. Maier (in Vorbereitung); Vorläufige Mitteilung: Z. Naturforschg. 13 a, 564 [1958].

7 P. Chatelain, Thèses, Paris 1937.
} 
während bei der statischen DK, wo die Anisotropie der Molekülumgebung vernachlässigt wurde ${ }^{1}$, die DK-Anisotropie nur wenige Prozent ausmacht. Wir verwenden daher Beziehungen, die von NeUgebauer ${ }^{8}$ für Kristalle mit anisotrop polarisierbaren Molekülen und nur einem Molekül in der Elementarzelle abgeleitet worden sind. Wir spezialisieren diese Beziehungen auf den Fall des optisch einachsigen Kristalls und erhalten:

$$
\begin{aligned}
\alpha_{1}^{*} & =\frac{n_{1}^{2}-1}{n_{1}^{2}+2-2 a\left(n_{1}^{2}-1\right)} \frac{M}{\varrho} \frac{3}{4 \pi N_{\mathrm{L}}} \\
\text { und } \quad \alpha_{\mathrm{q}}^{*} & =\frac{\bar{n}_{2}^{2}-1}{n_{2}^{2}+2+a\left(\bar{n}_{2}^{2}-1\right)} \frac{M}{\varrho} \frac{3}{4 \pi N_{\mathrm{L}}} .
\end{aligned}
$$

Es liegt nahe, als erste Näherung den Azoxyanisolkristall in dieser Weise zu behandeln, da die Moleküllängsachsen praktisch parallel zur Richtung des größten Brechungsindex $n_{1}$ liegen $^{7}$ und $n_{2}$ und $n_{3}$ sich nur wenig voneinander unterscheiden, so daß man näherungsweise den Kristall als optisch einachsig betrachten kann. Aus $n_{1}$ und $\bar{n}_{2}=\frac{1}{2}\left(n_{2}+n_{3}\right)$ lassen sich damit ohne weitere Zwischenrechnungen die frequenzabhängigen Längs- und Querpolarisierbarkeiten berechnen.

Zur Extrapolarisation $\operatorname{der} \alpha^{*}(\lambda)$-Werte auf $\lambda=\infty$ benutzen wir die Dispersionsformel

$$
\alpha^{*}=\text { const } /\left(v_{0}^{2}-v^{2}\right),
$$

die genügenden Frequenzabstand von der langwelligsten optischen Absorptionsbande voraussetzt, und bestimmen $\alpha^{*}(\infty)$ graphisch mit Hilfe der Geraden, die sich wegen

$$
\frac{1}{\alpha^{*}(\lambda)}=\frac{1}{\alpha^{*}(\infty)}+\text { const } / \lambda^{2}
$$

ergibt, wenn man $1 / \alpha^{*}(\lambda)$ gegen $1 / \lambda^{2}$ aufträgt.

Bei $\bar{\alpha}^{*}(\lambda)$ erhält man eine deutlich gekrümmte Kurve; offenbar ist man noch nicht weit genug vom ersten Elektronenübergang entfernt. Die Kurve läßt sich aber auf der langwelligen Seite ohne Zwang in einer Geraden fortsetzen, so daß man $\bar{\alpha}^{*}$ mit einiger Sicherheit bestimmen kann. Bei $\alpha_{\mathrm{q}}{ }^{*}(\lambda)$ liegen alle Werte auf einer Geraden, während $\alpha_{1}^{*}(\lambda)$ nicht in dieser Weise extrapoliert werden kann. Der Grund hierfür ist die ausgeprägte Polarisation des bei $\lambda=3600 \AA$ gelegenen $\pi-\pi$-Überganges, dessen Übergangsmoment parallel zur Moleküllängsachse

${ }^{8}$ H. E. J. Neugebauer, Canad. J. Phys. 32, 1 [1954].

9 W. Maier u. A. Saupe, Z. physikal. Chem. 6, 327 [1956].

10 K. v. Auwers u. P. Heimke, Ber. 61, 1030 u. 1037 [1928].

11 Landolt-Börnstein, Zahlenwerte und Funktionen, Band 1, Teil 3, p. 513 [1951]. orientiert ist ${ }^{9}$, so daß er bei $n_{1}$ sehr stark, bei $n_{2}$ und $n_{3}$ dagegen nur schwach wirksam ist.

Um die Gesamtpolarisierbarkeiten zu erhalten, addieren wir - wie üblich - 10\% der Elektronenpolarisierbarkeit $\alpha^{*}$ zu letzterer hinzu. Da kein Grund besteht, für die Atompolarisierbarkeiten eine ähnliche Anisotropie mit Bevorzugung der Längsachse anzunehmen wie bei $\alpha^{*}$, rechnen wir die $10 \%$ von $\bar{\alpha}^{*}$, also

$$
\bar{\alpha}=1,1 \bar{\alpha}^{*}, \quad \alpha_{1}=\alpha_{1}^{*}+0,1 \bar{\alpha}^{*}, \quad \alpha_{q}=\alpha_{q}^{*}+0,1 \bar{\alpha}^{*}
$$

und erhalten die in Tab. 1 angegebenen Werte.

\begin{tabular}{|c|cccc|}
\hline Elektronenpolarisierbarkeit & $\alpha^{*}$ & $\alpha_{1}{ }^{*}$ & $\alpha_{\mathrm{q}}{ }^{*}$ & $\Delta \alpha^{*}$ \\
$\alpha^{*}$ in $10^{-25} \mathrm{~cm}^{3}$ & 300 & 442 & 229 & 213 \\
\hline Gesamtpolarisierbarkeit & $\bar{\alpha}$ & $\alpha_{1}$ & $\alpha_{\mathrm{q}}$ & $\Delta \alpha$ \\
$\alpha$ in $10^{-25} \mathrm{~cm}^{3}$ & 330 & 472 & 259 & 213 \\
\hline
\end{tabular}

Tab. 1. p-Azoxyanisol-Molekül.

Zur Kontrolle berechnen wir $\bar{\alpha}^{*}$ noch auf andere Weise: Von der durch v. Auwers ${ }^{10}$ gemessenen Molrefraktion des 4-Äthoxy-azoxybenzols ausgehend erhält man mit den Werten $18,2 \cdot 10^{-25}$ und $22,6 \cdot 10^{-25}$ für die Elektronenpolarisierbarkeiten der $\mathrm{CH}_{2}$ - und der $\mathrm{CH}_{3}$-Gruppe ${ }^{11}$ die Molrefraktion des Azoxyanisols zu $R_{\infty}=75,6 \mathrm{~cm}^{3}$ und daraus $\bar{\alpha}^{*}=300 \cdot 10^{-25}$. Mit den tabellierten mittleren Bindungspolarisierbarkeiten ${ }^{11}$ ergibt sich $\bar{\alpha}^{*}=290 \cdot 10^{-25}$.

\section{c) Orientierung des Dipolmoments im Molekül}

Mit $\bar{\alpha}=330 \cdot 10^{-25}$ errechnet sich aus $\varepsilon_{\text {is }}$ nach (1) ein Dipolmoment vom Betrage $\mu=2,22 D$ (aus DKMessungen an verdünnten Lösungen in Benzol erhält man $\mu=2,30 D$ nach Messungen von Maier und Baumgartner ${ }^{12}$, ausgewertet mit $R_{\infty}=75,6$ ).

Für die Bestimmung des Momentwinkels $\beta$ aus $\Delta \varepsilon$ nach (4) benutzen wir den aus $\varepsilon_{\text {is }}$ errechneten $\mu$-Wert, um in beiden Fällen von Dielektrizitätskonstanten der reinen Flüssigkeit auszugehen. Man erhält, unabhängig von der Temperatur, $\beta=64^{\circ}$. Dieser Winkel ist von $Z_{\text {wetkoff }}$ und Marinin ${ }^{13}$ aus KERR-Effektmessungen an verdünnten Lösungen zu $\beta=57^{\circ} 40^{\prime}$ ermittelt worden. Die Autoren benutzten dabei für $\Delta \alpha$ den Wert $180 \cdot 10^{-25} \mathrm{~cm}^{3}$, der sich bei

12 W. Maier u. G. Baumgartner, Z. Naturforschg. 7 a, 172 [1952].

13 W. Zwetkow u. W. Marinin, J. exp. theor. Phys. 18, 641 [1948]. 
einer Auswertung nach der Lonentz-Formel aus den Chatelainschen Brechungsindizes ergibt. Wie oben schon erwähnt, ist das Lonentz-Feld für den stark anisotropen Kristall aber nicht brauchbar; die NEUGEBAUER-Beziehungen (6) stellen eine wesentlich bessere Näherung dar. Mit unserem $\Delta \alpha$ von $213 \cdot 10^{-25}$ und $\mu=2,30$ (Lösungswert!) erhält man aus der KeRR-Konstanten $K=57,8 \cdot 10^{12}$ den Winkel zu $\beta=61^{\circ}$, in ausreichender Übereinstimmung mit dem aus der dielektrischen Anisotropie berechneten Wert.

Aus $\beta$ läßt sich $\gamma$ berechnen, d. h. der Winkel, den das Teilmoment $\vec{\mu}_{\mathrm{M}}$ der Azoxygruppe mit der Verbindungslinie der Benzolringmittelpunkte des Azoxyanisolmoleküls bildet. Wir benutzen hierzu für $\mu_{\mathrm{M}}$ das Dipolmoment des Azoxybenzols $(=1,70 D)^{14}$, für $\sqrt{2} \cdot \mu_{\perp}$ das des Hydrochinondimethyläthers $(=1,67 D)^{15}$ und erhalten mit $\delta=11^{\circ}$ und $\mu=2,30$ für Azoxyanisol (Lösungswert, weil die anderen Dipolmomente ebenfalls an Benzollösungen bestimmt sind) den Wert $\gamma=54^{\circ}$.

\section{d) Nachweis, daß die gemessenen $\varepsilon_{1}$-Werte „statische“ Werte sind}

Die molekulare Theorie der DK nematischer Flüssigkeiten hatte ergeben ${ }^{1}$, daß in $\varepsilon_{1}$ ein Anteil von Orientierungspolarisation enthalten sein muß, der sich infolge der in der nematischen Phase besonders starken Behinderung des "180 $180^{\circ}$-Umklappens" der Moleküllängsachsen möglicherweise mit abnorm groBer Relaxationszeit einstellt. Es ist die Frage, ob das zugehörige $\varepsilon_{1}$-Dispersionsgebiet oberhalb oder unterhalb unserer Meßfrequenz liegt. Die beobachteten Andeutungen eines bei $1,6 \mathrm{MHz}$ beginnenden $\varepsilon_{1}$-Abfalls (s. oben) lassen ersteres vermuten. Danach sollten die bei 100,260 und $650 \mathrm{kHz}$ gemessenen $\varepsilon_{1}$-Werte noch die "statischen“ Werte sein. Dieser Schluß wird durch die nachfolgenden Überlegungen bestätigt.

Während Beziehung (2) den „statischen“ Wert $\left(\varepsilon_{1}\right)_{0}$ angibt, gilt für Frequenzen oberhalb des ersten $\varepsilon_{1}$-Dispersionsgebietes ${ }^{1}$

$$
\left(\varepsilon_{1}\right)_{\mathrm{I}}=\left(\varepsilon_{1}\right)_{0}-4 \pi N_{\mathrm{n}} h F^{2} \frac{\mu_{\mathrm{M}}^{2}}{3 k T} \cos ^{2} \gamma(1+2 S),
$$

wobei der zweite Term auf der rechten Seite den nun ausgefallenen Beitrag der Längskomponente des molekülfesten Teilmomentes $\mu_{\mathrm{M}}$ zu $\varepsilon_{1}$ darstellt. Wir

14 K. A. Gehrkens u. E. Müller, Liebigs Ann. Chem. 500, 296 [1933]. berechnen nun $\left(\varepsilon_{1}\right)_{0}$ nach $(2)$ und $\left(\varepsilon_{1}\right)_{\text {I }}$ nach (7) mit den bei $115^{\circ} \mathrm{C}$ experimentell bestimmten v-, $\bar{\varepsilon}$ - und $S$-Werten, sowie mit $\bar{\alpha}=330 \cdot 10^{-25}$, $\Delta \alpha=213 \cdot 10^{-25} \mathrm{~cm}^{3}$ und $\mu=2,22 D$ für verschiedene Werte des Winkels $\beta$, wobei alle $\beta$-Werte in Betracht zu ziehen sind, die sich mit $\mu_{\mathrm{M}}=1,70 \mathrm{D}$, $\sqrt{2} \mu_{\perp}=1,67 \mathrm{D}$ und $\delta=11^{\circ}$ für $\gamma$ zwischen 0 und $90^{\circ}$ ergeben. In Tab. 2 sind die so erhaltenen Werte zusammengestellt. Es wurde die tiefstmögliche Temperatur (unterkühlter Zustand) gewählt, weil hier der stärkste Unterschied und die größte Relaxationszeit zu erwarten sind.

\begin{tabular}{|r|c|c|c|}
\hline$\gamma$ & $\beta$ & $\left(\varepsilon_{1}\right)_{0}$ & $\left(\varepsilon_{1}\right)_{\text {I }}$ \\
\hline $0^{\circ}$ & $43^{\circ}$ & 7,57 & 3,91 \\
$54^{\circ}$ & $64^{\circ}$ & 5,87 & 4,62 \\
$90^{\circ}$ & $82^{\circ}$ & 4,99 & 4,99 \\
\hline
\end{tabular}

Tab. 2. $\varepsilon_{1}$-Dispersion bei $115^{\circ} \mathrm{C}$.

Der Vergleich mit dem experimentellen Wert $\varepsilon_{1}=5,628$ ergibt, daß $\left(\varepsilon_{1}\right)_{\text {I }}$ für keinen Winkel $\gamma$ bzw. $\beta$ auch nur annähernd mit dem Meßwert verträglich ist. Tatsächlich sind oberhalb des Relaxationsgebietes sogar noch kleinere Werte $\left(\varepsilon_{1}\right)_{\mathrm{I}}$ zu erwarten, als in Tab. 2 angegeben sind, da in (7) die Änderung des inneren Feldes beim Überschreiten des Relaxationsgebietes noch nicht berücksichtigt ist und da außerdem der Abfall der mittleren DK beim Übergang von der isotropen in die nematische Phase in den Formeln nicht enthalten ist.

Damit ist gezeigt, daß bei den verwendeten Meßfrequenzen das zu erwartende $\varepsilon_{1}$-Dispersionsgebiet noch nicht überschritten ist und die gemessenen Werte $\varepsilon_{1}$ dem statischen Fall entsprechen. Die Dispersionsstufe $\left(\varepsilon_{1}\right)_{0}-\left(\varepsilon_{1}\right)_{\text {I }}$ dürfte nach Tab. 2 (für $\gamma=54^{\circ}$ ) mindestens den Wert 1,2 haben.

\section{e) Verschiebungsanteil und Orientierungsanteil. Temperaturabhängigkeit}

Für die weitere Diskussion betrachten wir - um den direkten Dichteeinfluß zu eliminieren - die molaren Suszeptibilitäten

$$
\sigma=\frac{\varepsilon-1}{4 \pi} \frac{N_{\mathrm{L}}}{N}=\frac{\varepsilon-1}{4 \pi} \frac{M}{\varrho}
$$

und teilen sie jeweils in einen Polarisierbarkeitsanteil $\sigma_{\alpha}$ und einen Orientierungsanteil $\sigma_{\mu}$ auf. Aus

15 O. Hassel u. E. Naeshagen, Z. phys. Chem. B 8, 357 [1930]. 


\begin{tabular}{|c|c|c|c|c|c|c|c|c|c|c|}
\hline Phase & $t^{\circ} \mathrm{C}$ & $\varrho$ & $S$ & $\varepsilon$ & $F$ & $h$ & $\sigma_{\alpha}$ & $\sigma_{\mu}$ & $\sigma_{\text {ber }}$ & $\sigma_{\exp }$ \\
\hline $\begin{array}{l}\text { nematisch } \\
\text { (unterkühlt) }\end{array}$ & 115,0 & 1,1689 & 0,594 & $\begin{array}{l}\varepsilon_{1}=5,628 \\
\varepsilon_{2}=5,864\end{array}$ & 1,402 & 1,381 & $\begin{array}{l}48,32 \\
33,56\end{array}$ & $\begin{array}{l}37,34 \\
56,24\end{array}$ & $\begin{array}{l}85,66 \\
89,80\end{array}$ & $\begin{array}{l}81,37 \\
85,52\end{array}$ \\
\hline nematisch & 124,0 & 1,1606 & 0,540 & $\begin{array}{l}\varepsilon_{1}=5,602 \\
\varepsilon_{2}=5,784\end{array}$ & 1,397 & 1,380 & $\begin{array}{l}47,19 \\
33,84\end{array}$ & $\begin{array}{l}37,27 \\
53,92\end{array}$ & $\begin{array}{l}84,46 \\
87,76\end{array}$ & $\begin{array}{l}81,49 \\
84,72\end{array}$ \\
\hline nematisch & 133,0 & 1,1510 & 0,442 & $\begin{array}{l}\varepsilon_{1}=5,600 \\
\varepsilon_{2}=5,711\end{array}$ & 1,391 & 1,379 & $\begin{array}{l}45,35 \\
34,48\end{array}$ & $\begin{array}{l}38,06 \\
51,24\end{array}$ & $\begin{array}{l}83,41 \\
85,74\end{array}$ & $\begin{array}{l}82,14 \\
84,12\end{array}$ \\
\hline isotropflüssig & 135,4 & 1,1439 & 0,000 & $\varepsilon_{\text {is }}=5,702$ & 1,388 & 1,379 & 38,05 & 46,43 & 84,48 & 84,48 \\
\hline
\end{tabular}

Tab. 3. p-Azoxyanisol. Dielektrische Suszeptibilitäten.

\begin{tabular}{|c|c|c|c|c|c|}
\hline$t^{\circ} \mathrm{C}$ & $S$ & $\Delta \sigma_{\alpha}$ & $\Delta \sigma_{\mu}$ & $\Delta \sigma_{\text {ber }}$ & $\Delta \sigma_{\mathrm{e} x \mathbf{p}}$ \\
\hline 115,0 & 0,594 & $+14,75$ & $-18,90$ & $(-4,15)$ & $-4,15$ \\
124,0 & 0,540 & $+13,35$ & $-16,65$ & $-3,30$ & $-3,22$ \\
133,0 & 0,442 & $+10,87$ & $-13,20$ & $-2,33$ & $-1,98$ \\
\hline
\end{tabular}

Tab. 4. p-Azoxyanisol. Dielektrische Anisotropie.

(2) folgt z. B. für $\sigma_{1}$ :

$$
\begin{aligned}
& \sigma_{1 a}=N_{\mathrm{L}} h F\left(\bar{\alpha}+\frac{2}{3} \Delta \alpha \cdot S\right), \\
& \sigma_{1, u}=N_{\mathrm{L}} h F^{2} \frac{\mu^{2}}{3 k T}\left\{1-\left(1-3 \cos ^{2} \beta\right) S\right\} .
\end{aligned}
$$

Entsprechendes gilt für $\sigma_{\text {is }}, \sigma_{2}$ und $\Delta \sigma$.

Mit den aus den optischen Daten bestimmten Werten $\bar{\alpha}$ und $\Delta \alpha$ der Tab. 1 , dem aus der DK der isotropflüssigen Phase gewonnenen Dipolmoment $\mu$ (s. oben) und dem aus der DK-Anisotropie (bei $115^{\circ} \mathrm{C}$ ) errechneten Winkel $\beta$ bestimmen wir nun die aus den modifizierten Gln. (2) und (3) sich ergebenden Werte $\sigma_{a}$ und $\sigma_{\mu}$ für drei verschiedene Temperaturen der nematischen Phase. Tab. 3 bringt die hierzu verwendeten weiteren Daten und die Ergebnisse. Spalte 10 enthält die Summe $\sigma_{\alpha}+\sigma_{\mu}$, also die berechneten $\sigma$-Werte, Spalte 11 die aus $\varepsilon$ und $\varrho$ sich unmittelbar nach (8) ergebenden experimentellen $\sigma$-Werte.

Der $\sigma$-Wert der isotropflüssigen Phase $\left(135,4^{\circ}\right)$ ist ein Anschlußwert, da das benutzte $\mu$ aus dem $\varepsilon$ der isotropen Phase ermittelt wurde. Die $\sigma_{\text {ber- }}$-Werte der nematischen Phase liegen - wie Tab. 3 zeigt allgemein etwas höher als die experimentellen Werte; die Differenz steigt von ca. $2 \%$ bei $133^{\circ}$ auf ca. $5 \%$ bei $115^{\circ}$. Diese Diskrepanz entspricht der Tatsache, daß die auf den Klärpunkt extrapolierte gemessene mittlere Suszeptibilität der nematischen Phase um etwa $1,5 \%$ unter der aus der isotropflüssigen Phase extrapolierten liegt. Derartig kleine Diskrepanzen sind verständlich, da das der Theorie zugrunde lie- gende Modell, insbesondere der ONSAGER-Ansatz für das innere Feld, nicht alle Feinheiten richtig wiedergeben kann.

Tab. 3 zeigt, wie sich die Gesamtpolarisation aus einem Verschiebungsanteil mit stark positiver Anisotropie $\left(\sigma_{1 \alpha}>\sigma_{2 a}\right)$ und einem Orientierungsanteil mit stark negativer Anisotropie $\left(\sigma_{1 \mu}<\sigma_{2 \mu}\right)$ zusammensetzt. Die beobachtete DK-Anisotropie ist das Ergebnis dieser beiden sich entgegenwirkenden Beiträge.

Tab. 4 zeigt die Beiträge der beiden Polarisationsanteile zur dielektrischen Anisotropie. Man sieht, daß die Anisotropie der Verschiebungspolarisation und die der Orientierungspolarisation um nahezu den Faktor 5 größer sind als die Anisotropie der Gesamtpolarisation und sich zum großen Teil gegenseitig kompensieren. Natürlich vermag dementsprechend die Theorie nur noch angenähert richtige Werte für den Temperaturgang der Differenzgröße $\Delta \sigma \mathrm{zu}$ liefern. Der $115^{\circ}$.Wert ist der zur Bestimmung des Winkels $\beta$ benutzte Anschlußwert.

\section{Schluß}

Die einfache molekulare Theorie ${ }^{1}$ der dielektrischen Polarisation nematischer Phasen gibt, wie hier am Beispiel des Azoxyanisols gezeigt wurde, die dielektrischen Eigenschaften in ihren wesentlichen $\mathrm{Zü-}$ gen richtig wieder und zeigt insbesondere, wie die dielektrische Anisotropie durch die Überlagerung der beiden sich entgegen wirkenden Anisotropien 
der Verschiebungspolarisation und der Orientierungspolarisation zustande kommt. Das andersartige dielektrische Verhalten der Azobenzolderivate und das Verhalten der höheren Azoxyanisol-Homologen ist jetzt voll verständlich. Über entsprechende Untersuchungen an diesen kristallinflüssigen Substanzen wird in Kürze berichtet werden ${ }^{\mathbf{1 6}, 4}$. Wir haben in der hier vorliegenden Arbeit die Theorie benutzt, um aus den gemessenen DK-Werten eine molekulare Größe, nämlich das permanente Dipolmoment und seine Orientierung im Molekül, zu gewinnen. Der

16 W. MAIER u. G. MeIer (in Vorbereitung).
Erfolg beweist, daß es - umgekehrt - möglich ist, die Hauptwerte der statischen DK der nematischen Phase annähernd richtig zu berechnen, wenn die vollständigen elektrischen Daten des Moleküls und der nematische Ordnungsgrad gegeben sind (iteratives Verfahren wegen $h$ und $f$ ).

Die vorliegenden Untersuchungen wurden im Physikalischen Institut der Universität Freiburg/Br. durchgeführt, dessen Direktor, Herrn Prof. Tн. Sснміdт, wir für seine freundliche Unterstützung hiermit vielmals danken. Der Deu t schen Forschung sgemein$\mathrm{schaft}$ und dem Fonds der Chemie sind wir für großzügige finanzielle Beihilfe ebenfalls zu großem Dank verbunden.

\title{
Gruppentheoretische Betradhtungen zum Mikrowellenspektrum von Molekülen enthaltend zwei behindert drehbare Methylgruppen mit versdiedenen Kohlenstoffisotopen
}

\author{
Von H. Dreizler \\ Aus dem Institut für physikalische Chemie der Universität Freiburg \\ (Z. Naturforschg. 16 a, 477-484 [1961] ; eingegangen am 28. Januar 1961)
}

\begin{abstract}
Mit $\mathrm{C}^{12} \mathrm{H}_{3} \mathrm{SC}^{13} \mathrm{H}_{3}$ wird ein Typ eines asymmetrischen Moleküls mit zwei behindert drehbaren, symmetrischen Molekülgruppen untersucht. Das Rotationsspektrum dieses Molekültyps muß nach gruppentheoretischen Überlegungen Unterschiede gegenüber dem ebenfalls durch behinderte Rotation modifizierten Rotationsspektrum eines Moleküls etwa vom Typ des $\left(\mathrm{CH}_{3}\right)_{2} \mathrm{CO}$ aufweisen. Ausgehend vom exakten Hamilton-Operator, der invariant gegen eine Gruppe von 18 Symmetrieoperationen ist, werden der Entartungsgrad der Eigenwerte und die Auswahlregeln angegeben. Weiter werden über einen vereinfachten, näherungsweise gültigen Hamiston-Operator die Aufspaltungsmuster der Rotationslinien, im äußersten Falle Quintetts, und die relativen Intensitäten der Komponenten der Multipletts abgeleitet. Die verwendeten Symmetriegruppen werden erläutert.
\end{abstract}

Eine vollständige Strukturanalyse eines Moleküls vom Typ des Dimethylsulfids ${ }^{1}$, das als Beispiel gewählt wird, des Dimethyläthers ${ }^{2}$ oder Acetons ${ }^{3}$ mit dreizähliger Symmetrie der drehbaren Gruppen verlangt die Untersuchung einer Reihe von isotopen Verbindungen. Dabei kommt der Kohlenstoffisotopie besondere Bedeutung zu, weil die Ersetzung der Hdurch D-Atome infolge wesentlicher Änderung der Nullpunktsschwingungsamplituden merkliche Fehler in den Strukturparametern verursacht, die bei der $\mathrm{C}^{\mathbf{1 3}}$-Substitution sicher weitaus kleiner sind. Da im allgemeinen die einfach $\mathrm{C}^{13}$-substituierten Moleküle viel häufiger auftreten als die zweifach substituierten, wird man auch sie zweckmäßig zur Struktur-

1 H. D. Rudolph, H. Dreizler u. W. Maier, Z. Naturforschg. 15 a, 742 [1960].

2 P. H. Kasai u. R. J. Myers, J. Chem. Phys. 30, 1096 [1959].

3 J. D. Swalen u. C. C. Costain, J. Chem. Phys. 31, 1562 [1959]. aufklärung heranziehen, obwohl die einfache Substitution gewisse Komplikationen mit sich bringt, indem sich das Spektrum hinsichtlich des Aufspaltungsmusters der einzelnen Linien verändert, und weitere, wenn auch schwache Linien durch a-Übergänge erzeugt werden. Dies veranlaßte uns, allgemeine Überlegungen über den Aufbau des Rotationsspektrums von $\mathrm{C}^{12} \mathrm{H}_{3} \mathrm{SC}^{13} \mathrm{H}_{3}$ anzustellen. Das Spektrum dieses asymmetrischen Kreiselmoleküls ist wie bei allen derartigen Molekülen durch den Einfluß der beiden gehindert drehbaren Methylgruppen modifiziert. Gegenüber $\mathrm{C}^{12} \mathrm{H}_{3} \mathrm{SC}^{12} \mathrm{H}_{3}$, das eine $\mathrm{C}_{2}$-Konfigurationssymmetrie ${ }^{4}$ besitzt, ist die Konfigurationssymmetrie des einfach $\mathrm{C}^{\mathbf{1 3}}$-substituierten Moleküls je nach der

${ }^{4}$ Private Mitteilung von Prof. R. J. Myers über Symmetriefeststellung bei $\left(\mathrm{CH}_{3}\right)_{2} \mathrm{O}$ lassen bei $\left(\mathrm{CH}_{3}\right)_{2} \mathrm{~S}$ gleiche Verhältnisse erwarten. 